\title{
ELECTRICAL CHARGING OF UNCHARGED AEROSOL PARTICLES UNDER AT BIPOLAR ION CONCENTRATIONS
}

\author{
MOTOAKI ADACHI, KIKUO OKUYAMA, YASUO KOUSAKA \\ and TAKESHI TAKAHASHI \\ Radiation Center of Osaka Prefecture, Sakai 593
}

\begin{abstract}
The electrical charging process in which uncharged aerosol particles are mixed with various concentrations of bipolar ion was studied by solving the basic equation, the so-called birth-death equation, numerically for various conditions. Some of the calculated results were compared with the experimental results obtained by the visible experimental technique. As a result, the timedependent change in charge distribution of aerosol particles was found to be very rapid, and the equilibrium charge, which was effected by the ratio of number concentration of ion pairs to that of aerosols, was evaluated. The critical ratio where Boltzmann's equilibrium distribution was attained was also evaluated.
\end{abstract}

\section{Introduction}

Electrical charging of aerosol particles by bipolar ions is important in identifying the electrical property of atmospheric aerosols, especially the electrical conductivity of the atmosphere, which is used as an index of atmospheric pollution. The charging process is also important in connection with electrostatic particle-size analysis, the principle of which is based upon Boltzmann's equilibrium charge distribution.

The charging process at a high concentration of ion pairs compared with aerosol particle concentration has been studied theoretically by $\mathrm{Gunn}^{3)}$, Keefe et $a l^{4)}$, Whitby and Peterson ${ }^{12}$ and Takahashi' ${ }^{10)}$, and experimentally by Woessner and Gunn ${ }^{13)}$, Pollak and Metnieks ${ }^{8}$, and Liu and $\mathrm{Pui}^{5,6)}$. The charging process at a low concentration of bipolar ion, on the other hand, has only been studied by Pollak and Metnieks ${ }^{9)}$, Takahashi and Kudo ${ }^{11}$ and Marlow and Brock ${ }^{7}$. Pollak and Metnieks studied experimentally the charge equilibrium during aging of particles. Their experiment, however, seems to be inaccurate because of the existence of charged condensation nuclei produced by ionizing radiation and also because of the uncertainty of ion pair concentration. Takahashi and Kudo have studied the charging process in flow-type charging vessels from both theoretical and experimental points of view. But their analysis and experiments seem to have been done under very limited conditions. Though

Received February 28,1979. Correspondence concerning this article should be addressed to M. Adachi. K. Okuyama and Y. Kousaka are at Dept. of Chem. Eng., Univ. of Osaka Pref., Sakai 591. T. Takahashi is at Dept. of Nuclear Eng., Nagoya Univ., Nagoya 464.
Marlow and Brock have given the results of theoretical calculation considering the difference of physical properties between negative and positive ions, their theory has not been examined yet.

In this paper, the charging process in which uncharged particles are mixed with various concentrations of ion pairs, which will give the basic idea for understanding the charging process by bipolar ions, has been studied from both theoretical and experimental points of view.

\section{Theoretical Consideration}

\section{1 Basic equation}

The system shown in Fig. 1, where aerosol particles and ion pairs are mixed together in a mixing chamber, is analysed in this paper. The mixing of an aerosol with ion pairs is assumed to take place instantaneously in the mixing chamber, and the physical properties and the number of positive ions are assumed to be the same as those of negative ions. The "birth-death" equations for bipolar charging can be written as

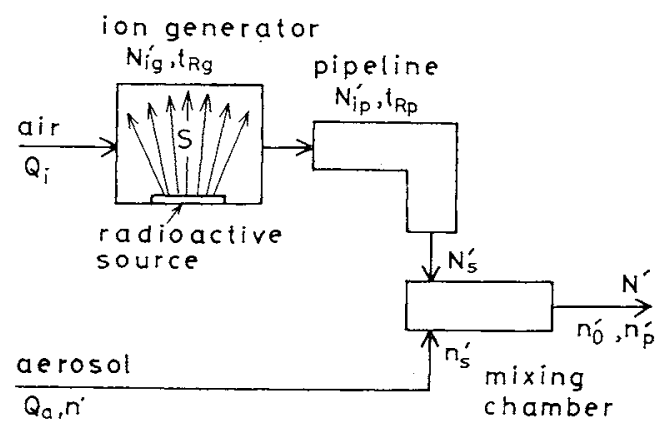

Fig. 1 Schematic diagram of system to be analysed 


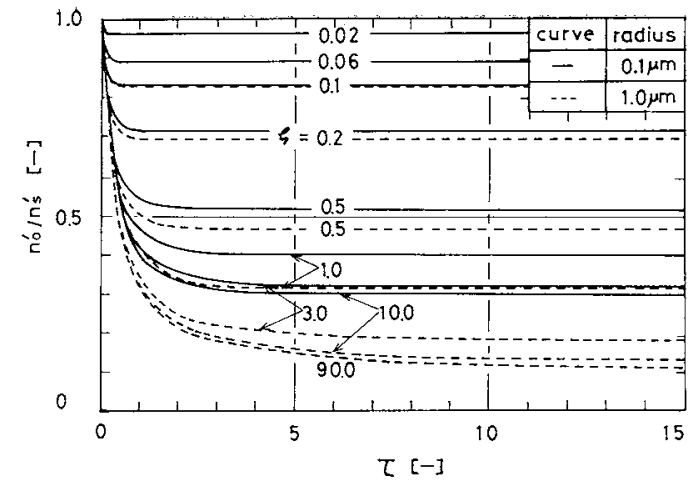

Fig. 2 Effect of $\zeta$ on rate of uncharged particle

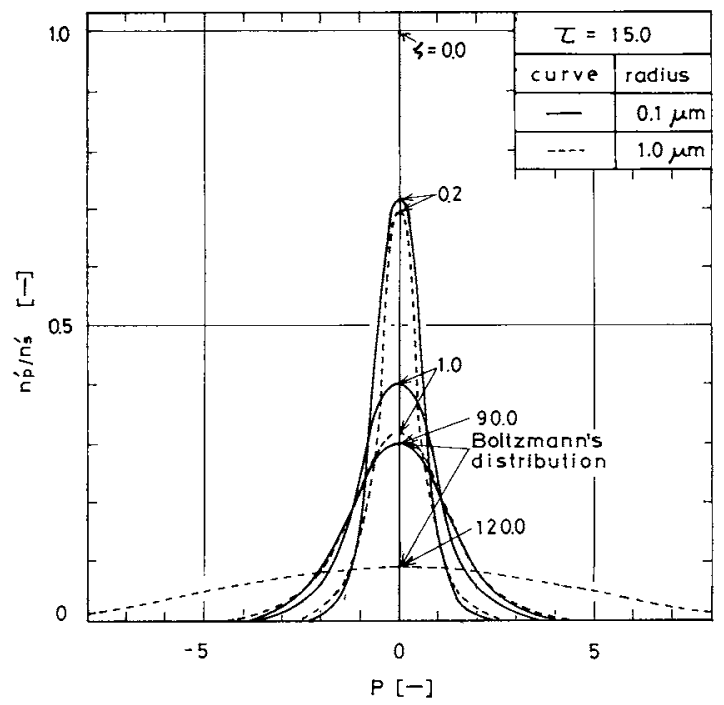

Fig. 3 Effect of $\zeta$ on electrical distribution

$$
\begin{aligned}
& d N^{\prime} / d t=-\alpha N^{\prime 2}-\left[U_{0}^{\prime} n_{0}^{\prime}+\sum_{p=1}^{\infty}\left(U_{p}^{\prime}+B_{p}^{\prime}\right) n_{p}^{\prime}\right] N^{\prime} \\
& d n_{0}^{\prime} / d t=\left(-2 U_{0}^{\prime} n_{0}^{\prime}+2 B_{1}^{\prime} n_{1}^{\prime}\right) N^{\prime} \\
& d n_{p}^{\prime} / d t=\left[U_{p-1}^{\prime} n_{p-1}^{\prime}-\left(U_{p}^{\prime}+B_{p}^{\prime}\right) n_{p}^{\prime}+B_{p+1}^{\prime} n_{p+1}^{\prime}\right] N^{\prime} \\
& |p| \geqq 1
\end{aligned}
$$

The left side of Eq. (1) represents the change in ion concentration $N^{\prime}$ with time $t$. The first term of the right side indicates the dissipation rate due to recombination of ions and the second term the loss rate of ions due to collision with particles. Equations (2) and (3) indicate the changes in number concentration of uncharged and charged particles with time. The first and third terms in brackets of the right side of Eq. (3) are the rate of appearance of particles carrying $p$ elementary unit of charge due to collisions of particles carrying $p-1$ unit of charge with same ions and collisions of particles carrying $p+1$ unit of charge with the opposite ions, and the second term is the rate of disappearance of these particles due to collisions with positive and negative ions. $U_{0}^{\prime}$ is the combination probability per ion with uncharged particles, and $U_{p}^{\prime}$ or $B_{p}^{\prime}$ indicates the combination probability per ion with charged particles which are carrying $p$ charges of the same sign or opposite sign, and they are given by ${ }^{23}$

$$
\begin{aligned}
& U_{0}^{\prime}=4 \pi D r /\left[4 D / r G_{i}+r /\left(r+l_{i}\right)\right] \\
& U_{p}^{\prime}=U_{0}^{\prime} y /[\exp (y)-1] \\
& B_{p}^{\prime}=U_{0}^{\prime} y /[1-\exp (-y)] \\
& y=p e^{2} / r \kappa T
\end{aligned}
$$

In dimensionless form, Eqs. (1), (2) and (3) can be written as

$$
\begin{aligned}
& d N / d \tau=-\left(\alpha / U_{0}^{\prime}\right) N^{2}-\left[n_{0}+\sum_{p=1}^{\infty}\left(U_{p}+B_{p}\right) n_{p}\right] N / \zeta \\
& d n_{0} / d \tau=\left(-2 n_{0}+2 B_{1} n_{1}\right) N \\
& d n_{p} / d \tau=\left[U_{p-1} n_{p-1}-\left(U_{p}+B_{p}\right) n_{p}+B_{p+1} n_{p+1}\right] N
\end{aligned}
$$

where $N=N^{\prime} / N_{s}^{\prime}$ ( $N_{s}^{\prime}$ is the initial ion pair concentration in mixing chamber), $n_{0}=n_{0}^{\prime} / n_{s}^{\prime}, n_{p}=n_{p}^{\prime} / n_{s}^{\prime}\left(n_{s}^{\prime}\right.$ is number concentration of particles in mixing chamber), $U_{p}=U_{p}^{\prime} / U_{0}^{\prime}, B_{p}=B_{p}^{\prime} / U_{0}^{\prime}, \tau=n_{s}^{\prime} U_{0}^{\prime} t$ and $\zeta=N_{s}^{\prime} / n_{s}^{\prime}$. As seen from Eqs. (8), (9) and (10), the solution of these equations depends mainly on the values of $\alpha / U_{0}^{\prime}$ and $\zeta$.

Initial ion pair concentration $N_{s}^{\prime}$ in mixing chamber required to solve the above equations is given as follows:

1) Change in ion pair concentration $N_{i g}^{\prime}$ in the ion generator can be expressed, when the dimension of the ion generator is nearly equal to the $\alpha$-ray range of the radioactive source ${ }^{1)}$, as

$$
d N_{i g}^{\prime} / d t=-\alpha N_{i g}^{\prime 2}-N_{i g}^{\prime} / t_{R g}+S
$$

The average retention time of air in the ion generator, $t_{R g}$, is assumed to be given by $V / Q_{i}$. Since the ion pair concentration $N_{i g}^{\prime}$ immediately attains the equilibrium state, the ion pair concentration in the ion generator is given as

$$
N_{i g}^{\prime}=\left(-1+\sqrt{\overline{1}+4 \alpha \overline{S t_{R g}^{2}}}\right) / 2 \alpha t_{R g}
$$

2) Assuming that the collision of ion onto the pipe wall is ignored, ion pair concentration $N_{i p}^{\prime}$ in a pipeline can be obtained by

$$
d N_{i p}^{\prime} / d t=-\alpha N_{i p}^{\prime 2}
$$

This equation can be solved analytically with the initial condition, $N_{i p}^{\prime}=N_{i g}^{\prime}$, to give

$$
N_{i p}^{\prime}=N_{i g}^{\prime} /\left(\alpha t_{R p} N_{i g}^{\prime}+1\right)
$$

3) Initial ion pair concentration $N_{s}^{\prime}$ and number concentration of particles $n_{s}^{\prime}$ in the mixing chamber are given respectively as

$$
N_{s}^{\prime}=N_{i p}^{\prime} Q_{i} /\left(Q_{i}+Q_{a}\right), n_{s}^{\prime}=n^{\prime} Q_{a} /\left(Q_{i}+Q_{a}\right)
$$

\section{2 Calculation method and results}

Since Eqs. (8), (9) and (10) cannot be solved analytically, these differential equations were solved numerically using the Runge-Kutta-Merson method. It was assumed for initial conditions that aerosol particles are all neutral and that the mixing chamber is uniformly filled with particles and bipolar ions, i. e. at $\tau=0.0 ; \quad N=1.0, \quad n_{0}=1.0, \quad n_{p}=0.0$ for $1 \leq|p| \leq 39$. The validity of the range of $p, 1 \leq|p| \leq 39$, was con- 


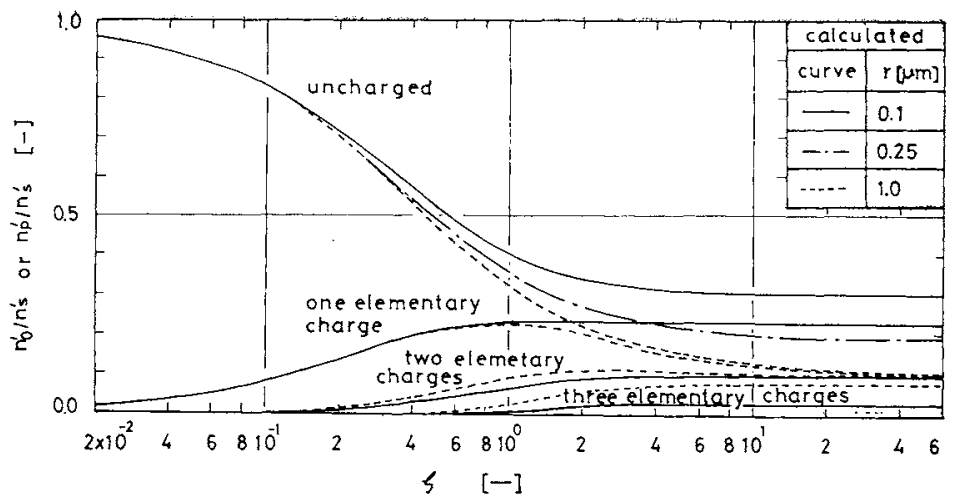

Fig. 4 Charge of uncharged or charged particle number fraction with $\boldsymbol{\zeta}$ firmed by comparing the calculation results when $1 \leq|p| \leq 39$ with those using a larger range of $p$, $1 \leq|p| \leq 150$.

To examine the effect of particle size $r$ and $\zeta$, calculations were made for values of $r$ from $0.1 \mu \mathrm{m}$ to $1 \mu \mathrm{m}$ and $\zeta$ from 0.01 to 100 . Figure 2 shows, as an example of the calculation results, the effect of $\zeta$ on the ratio of number concentration of uncharged particles to that of all particles, $n_{0}^{\prime} / n_{s}^{\prime}$. For a wide range of $\zeta$, the value of uncharged particle fraction seem to attain the equilibrium state at $\tau=15$. As the particle radius or the value of $\zeta$ increases, the time necessary to attain the equilibrium state tends to increase.

The equilibrium charge distributions for various values of $\zeta$ are shown in Fig. 3. It is seen that they approach the Boltzmann distributions at larger values of $\zeta$, which are given by the equation

$$
n_{p}^{\prime} / n_{s}^{\prime}=\exp \left(-p^{2} e^{2} / 2 r \kappa T\right) /\left[1+2 \sum_{p=1}^{\infty} \exp \left(-p^{2} e^{2} / 2 r \kappa T\right)\right]
$$

Figure 4 shows the change in the ratios of uncharged and charged particle number concentration to total particle number concentration with $\zeta$. The lines running almost parallel with the abscissa coincide with those of the Boltzmann distribution. As is seen in the figure the value of $\zeta$ required to attain the Boltzmann distribution increases with particle radius. In the case of $\zeta<0.1$, however, the charge distributions are independent of particle radius. This is because the number of ion pairs is controlling in the case of a very small number of ion pairs.

\section{Experimental Apparatus and Method}

The schematic diagram of the experimental apparatus is shown in Fig. 5 (a). The uncharged polydisperse DOP aerosol was produced by a generator as shown in Fig. 5(c). As seen from Fig. 5(c), no discharging device for condensation nuclei was used to generate uncharged particles. The change in number concentration and radius of aerosol particles during the experiment were kept within $\pm 5 \%$ by means of exact temperature control of boiler and reheater. The aerosol particles were not monodisperse because there were no condensation nuclei, but were thoroughly neutral. The particle-size spectrum was measured at the beginning and the end of experiment by the ultramicroscopic technique ${ }^{14}$.

Bipolar ions were produced by $\alpha$-ray from a Am-241 source. The Am-241 source was attached to the bottom of a channel of $4 \mathrm{~cm}$ depth to fit the $\alpha$-ray range, as shown in Fig. 5 (d). Two Am-241 radioactive sources, $100 \mu \mathrm{Ci}$ and $10 \mu \mathrm{Ci}$ of activity respectively, were used. The current between the bottom where Am-241 was attached and an electrode on the opposite channel wall was measured by an electrometer as a function of applied voltage. The current is seen to increase with voltage and to attain the saturation state. The ion pair production rate $S$ in unit volume of the ion generator is given by the saturation current, $I_{s}$, and the volume of ion generator, $V$, as, $S=I_{s} / e \cdot V$. The ion concentration was changed from $1.96 \times 10^{11}$ ion pairs $/ \mathrm{m}^{3}$ to $9.45 \times 10^{12}$ ion pairs $/ \mathrm{m}^{3}$ by changing the activity of radioactive source, flow rate of carrier air of ion and the length of pipeline. The pipeline is made of copper.

Aerosol particles and bipolar ions thus generated were mixed in a mixing chamber as shown in Fig. 5 (a). The mixing chamber, having a volume of $1.40 \times$ $10^{-4} \mathrm{~m}^{3}$, is made of copper and connected to a ground line. After aerosol particles mixed with bipolar ions were retained for a dimensionless time longer than $\tau=15$, they were introduced into the observation cell to detect charged particles. The observation cell was equipped with vertical parallel-plate electrodes as shown in Fig. 5 (b). The aerosol flow was instantaneously stopped by closing the valves after introducing an aerosol into the cell, then the aerosol inside the cell started to sedimentate. As the focus of the microscope is set at a certain position inside the cell to which a laser beam is applied, the aerosol particles existing in the sensing volume of the microscope are recognized because of light scattering. The particle images were photographed or recorded by a video recorder. Application of a certain voltage to the electrodes which compose the vertical side-wall of the cell causes aerosol particles to move toward one of the electrodes ac- 


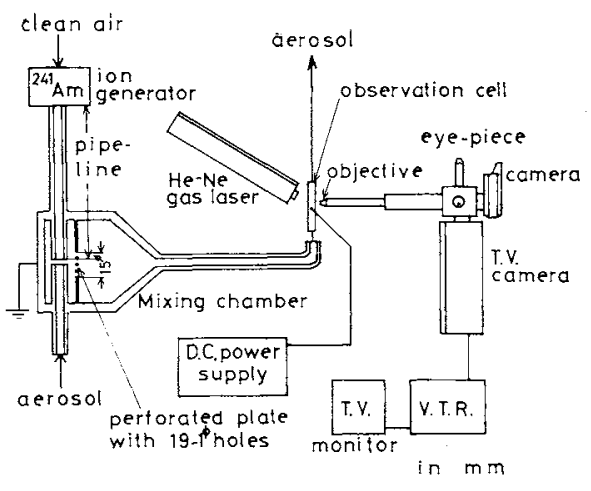

(a) Schematic diagram of experimental apparatus

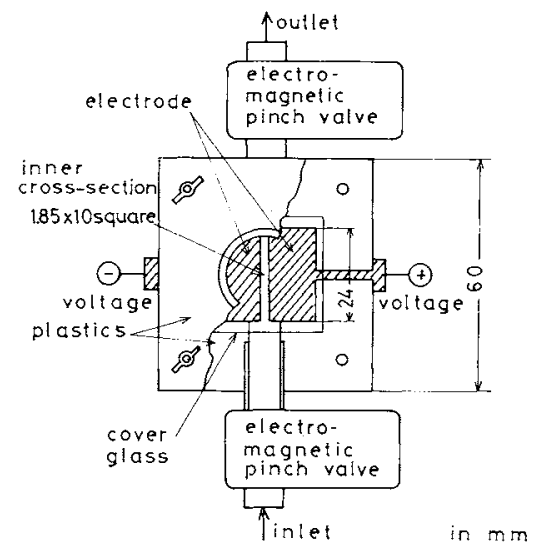

(b) Observation cell

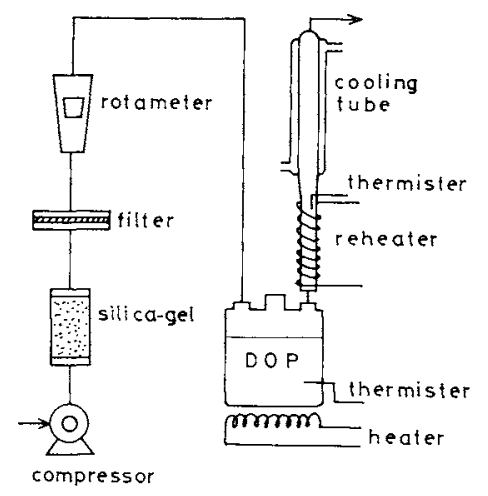

(c) Aerosal generator

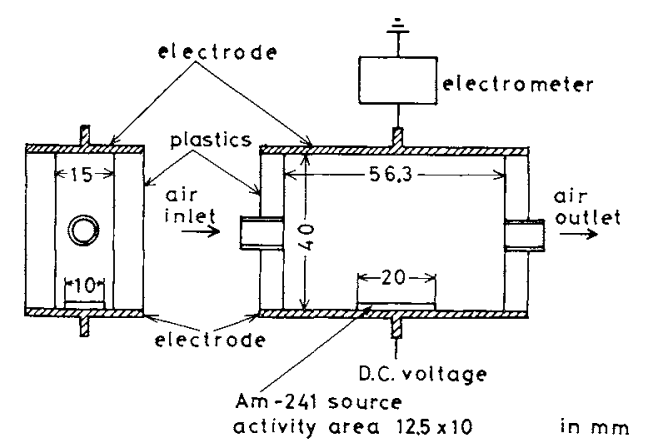

(d) Ion generator

Fig. 5 Experimental apparatus

\section{Table 1 Experimental conditions}

Aerosol

Kind of aerosol: di-octylphthalate (DOP)

Geometric mean radius: $r=0.360 .96 \mu \mathrm{m}$

Geometric standard deviation: $\sigma_{g}=1.35-1.54$

Particle number concentration:

$n^{\prime}=2.20 \times 10^{12}-4.10 \times 10^{12}$ particles $/ \mathrm{m}^{3}$

Ion

Flow rate: $Q_{a}=1.67 \times 10^{-5} \mathrm{~m}^{3} / \mathrm{sec}$

1) Ion generator

Radioactive source: $100 \mu \mathrm{Ci}$ and $10 \mu \mathrm{Ci} \mathrm{Am}-241$

Saturation current: $I_{s}=1.35 \times 10^{-8} \mathrm{~A}$ and $5.80 \times 10^{-10} \mathrm{~A}$

Ion pair production rate:

$\mathrm{S}=2.50 \times 10^{15}$ and $1.07 \times 10^{14}$ ion $\mathrm{pair} / \mathrm{m}^{3} \mathrm{sec}$

Volume: $\mathrm{V}=3.38 \times 10^{-5} \mathrm{~m}^{3}$

Retention time: $t_{R g}=0.68-2.03 \mathrm{sec}$

Flow rate: $Q_{i}=1.67 \times 10^{-5}-5.00 \times 10^{-5} \mathrm{~m}^{3} / \mathrm{sec}$

2) Pipeline

Cross-section area: $1.96 \times 10^{-5}$ and $5.0 \times 10^{-5} \mathrm{~m}^{2}$

Length: $L=0.09-0.525 \mathrm{~m}$

Retention time: $t_{R p}=0.53-1.58 \mathrm{sec}$

3) Mixing chamber

Volume: $1.40 \times 10^{-4} \mathrm{~m}^{3}$

Retention time: $2.1-4.2 \mathrm{sec}$

Flow rate: $Q_{a}+Q_{i}=3.33 \times 10^{-5}-6.67 \times 10^{-5} \mathrm{~m}^{8} / \mathrm{sec}$

Rate of ion pair concentration to particle concentration: $\zeta=9.60 \times 10^{-2}-1.72 \times 10^{1}$

Initial ion pair concentration: $N_{s}{ }^{\prime}=1.96 \times 10^{11}-9.45 \times 10^{12}$ ion pairs $/ \mathrm{m}^{3}$

cording to the sign of charged particles. The particle number $n_{s}^{\prime}$ in the observation cell is counted before application of voltage and then the uncharged particle number $n_{0}^{\prime}$ remaining in the cell is counted after voltage is applied to remove all of the charged particles. These observations were repeated until the sum of $n_{s}^{\prime}$ reached to about one thousand, a sufficient number in this experiment. Thus, the ratios $n_{0}^{\prime} / n_{s}^{\prime}$ of uncharged particle number to that of all particles were obtained for various ratios of ion concentration to particle concentration. Experimental conditions are shown in Table 1.

\section{Experimental Results and Discussion}

Cumulative size distributions of the aerosol particles used in the experiment are shown in Fig. 6.

Figure 7 shows the change in $n_{0}^{\prime} / n_{s}^{\prime}$ with ion pair concentration at the outlet of the pipeline, where ion pair concentration $N_{i p}^{\prime}$ at the outlet of the pipeline was calculated by Eq. (14), changing pipe length or retention time. The good agreement of experimental results with theoretical curves suggests the validity of Eq. (14). In this comparison, aerosols are assumed to be monodisperse, having the geometric mean radius as shown in Fig. 6.

Figure 8 shows a comparison of experimental results with corresponding theoretical curves. In this comparison, the experimental values decrease slightly more slowly than do the theoretical curves. This may be caused by the decrease in ion concentration due to deposition onto the pipe wall, which is not 

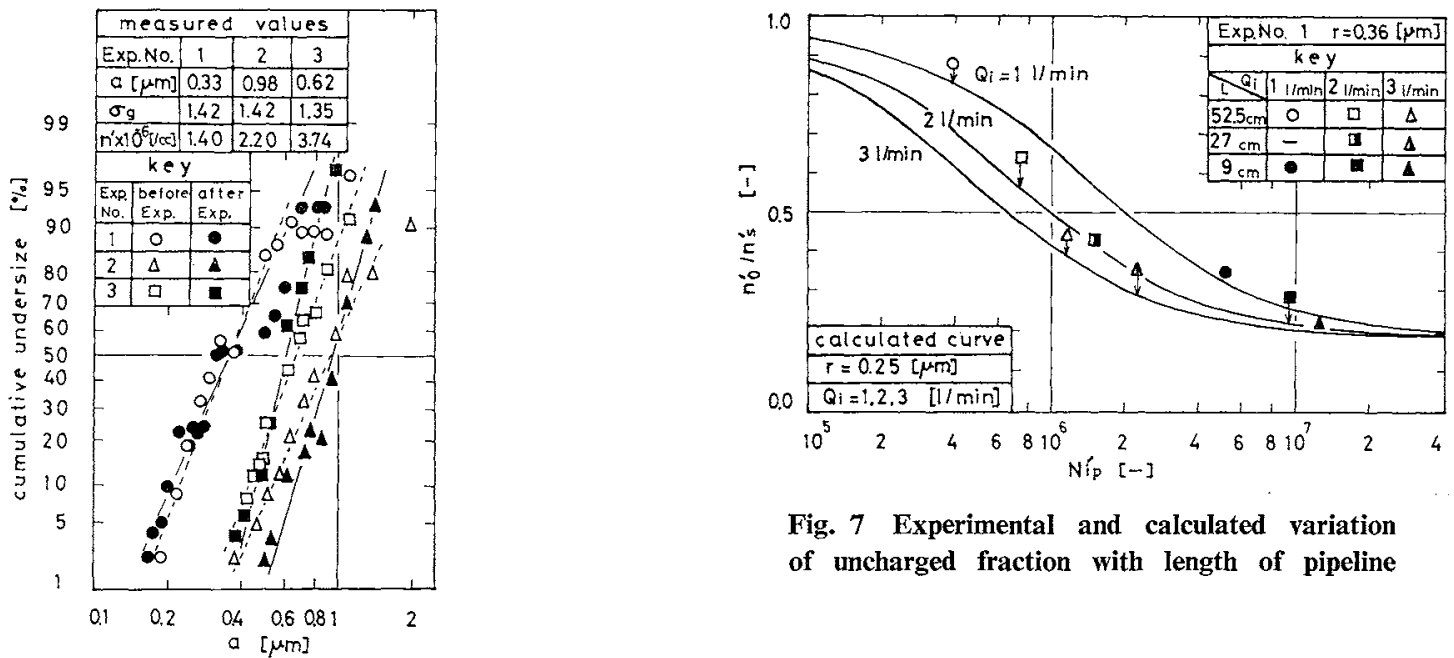

Fig. 7 Experimental and calculated variation of uncharged fraction with length of pipeline

Fig. 6 Cumulative size distribution of aerosols

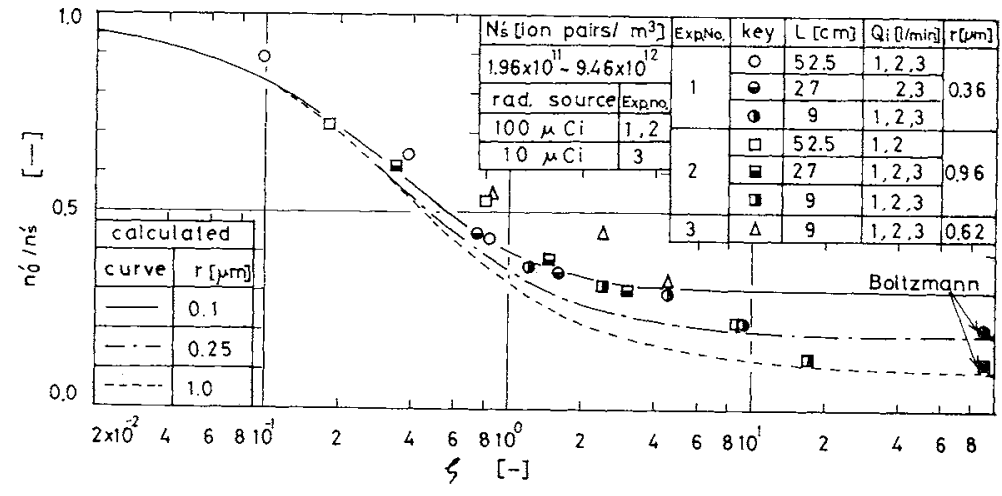

$(\bullet, \boldsymbol{\Xi})=$ experimental values when aerosol was directly passed through ion generator

Fig. 8 Comparison of experimental results with theoretical curves considered in our calculation in order to avoid complication. No difference between $0.36 \mu \mathrm{m}$ and 0.96 $\mu \mathrm{m}$ particle radius is found. When an aerosol was directly passed through the ion generator, the experimental values shown by the keys and in Fig. 8 are in good agreement with corresponding Boltzmann equilibrium values. Though the effect of polydispersity of aerosol on the charging process was not considered in the present calculation and though physical properties of ions were not directly measured by experiment, the overall agreement obtained in this graph suggests the validity of the analysis presented in this paper.

\section{Conclusion}

Conclusions drawn in this study on the charging of aerosol particles by bipolar ion are as follows.

1) The ratio of number concentration of ion pairs to that of aerosol particles, $\zeta$, which is proposed to generalize numerical calculations, plays an important role in understanding the diffusion charging of aerosol particles. The equilibrium charge under various number concentrations of ions and aerosols can be evaluated by using the ratio $\zeta$.

2) The existance of a critical ratio of $\zeta$ where the Boltzmann equilibrium distribution is attained is suggested.

3) The time required to attain an equilibrium charging state under various charging conditions is very short.

4) Using the visible experimental technique for observing charged and uncharged particles, satisfactory agreement between experiment and calculation was found.

$$
\begin{aligned}
& \text { Nomenclature } \\
& B_{p} \quad=\text { dimensionless combination probability } \\
& B_{p^{\prime}}=\text { combination probability per ion with } \\
& D \quad=\text { diffusion constant of ion }\left(=3.6 \times 10^{-8}\right) \quad\left[\mathrm{m}^{2} / \mathrm{sec}\right] \\
& e \quad=\text { elementary electrical charge } \\
& \left(=1.6019 \times 10^{-19}\right) \\
& G_{i} \quad=\text { mean thermal velocity of ion } \\
& \left(=4.50 \times 10^{2}\right) \\
& I_{s}=\text { saturation current } \\
& l_{i}=\text { mean free path of ion } \\
& \left(=1.3 \times 10^{-8}\right) \\
& N=\text { dimensionless ion pair concentration } \\
& \left(=N^{\prime} / N_{s}{ }^{\prime}\right) \\
& N^{\prime} \quad=\text { ion pair concentration in mixing } \\
& \text { chamber } \\
& =\text { initial ion pair concentration in mixing }
\end{aligned}
$$


chamber $\left(=N_{i j}{ }^{\prime} Q_{i} /\left(Q_{i}+Q_{a}\right) \quad\right.$ [ion pairs $\left./ \mathrm{m}^{3}\right]$

$=$ dimensionless particle number concentration $\left(=n^{\prime} / n_{s}{ }^{\prime}\right)$

$U_{p} \quad=$ dimensionless combination probability

$=$ particle number concentration [particles $\left./ \mathrm{m}^{3}\right]$

$=$ initial particle number concentration in mixing chamber $\left(=n^{\prime} Q_{a} /\left(Q_{i}+Q_{a}\right)\right)$ [particles $/ \mathrm{m}^{3}$ ]

$=$ number of elementary charge

$=$ flow rate of aerosol and ion

$=$ particle radius

$=$ ion pair production rate in unit volume of ion generator [ion pairs $/ \mathrm{m}^{3} \cdot \mathrm{sec}$ ]

$=$ absolute temperature

$=$ time

$=$ retention time in ion generator and pipeline per ion with particles carring $p$ charges of the same sign $\left(=U_{p}{ }^{\prime} / U_{0}{ }^{\prime}\right)$

$U_{0}{ }^{\prime}=$ combination probability per ion with uncharged particles

$U_{p}^{\prime} \quad=$ combination probability per ion with particles carrying $p$ charges of the same sign

$V$

$=$ volume of ion generator

$\left[\mathrm{m}^{3} / \mathrm{sec}\right]$

$=$ recombination constant of ion pairs $\left(=1.6 \times 10^{-12}\right)$

$=$ Boltzmann's constant $\left(=1.3806 \times 10^{-23}\right)$

$=$ geometric standard deviation

$=$ dimensionless time $\left(=n_{s}{ }^{\prime} U_{0}{ }^{\prime} t\right)$

$=$ ratio of ion pair concentration to particle number concentration $\left(=N_{s}{ }^{\prime} / n_{s}{ }^{\prime}\right)$ [ion pairs/particles]

〈Subscripts〉

ig $\quad=$ in ion generator

ip $\quad=$ in pipeline

$p \quad=p$ elementary charged

$0 \quad=$ uncharged

\section{Literature Cited}

1) Cooper, D. W. and R. C. Reist: J. Colloid Interface Sci., 45, 17 (1973).

2) Fuchs, N. A. and A. G. Sutugin: "Topies in Current Aerosol Research (F. M. Hidy and J. R. Brock ed.)", p. 44, Pergamon, Oxford (1971).

3) Gunn, R.: J. Metrorl., 11, 339 (1954).

4) Keefe, D., P. J. Nolan and T. A. Rich: Proc. Roy. Irish Acad., 60A, 27 (1959).

5) Liu, B. Y. H. and D. Y. H. Pui: J. Aerosol Sci., 5, 465 (1974).

6) Liu, B. Y. H. and D. Y. H. Pui: J. Colloid Interface Sci., 47, 305 (1974).

7) Marlow, W. H. and J. R. Brock: ibid., 51, 23 (1975).

8) Pollak, L. W. and A. L. Metniecks: Geof. Pure. Appl., 51, 225 (1962).

9) Pollak, L. W. and A. L. Metniecks: ibid., 53, 111 (1962).

10) Takahashi, K: J. Colloid Interface Sci., 35, 508 (1971).

11) Takahashi, K. and A. Kudo: J. Aerosol Sci., 4, 209 (1973),

12) Whitby, K. T. and C. N. Peterson: Ind. Eng. Chem., Fundam., 4, 66 (1965).

13) Woessner, R. H. and R. Gunn: J. Colloid Interface Sci., 11, 69 (1956).

14) Yoshida, T., Y. Kousaka and K. Okuyama: Ind. Eng. Chem., Fundam., 14, 47 (1975). 